Website: http://jsci.utq.edu.iq

Email: utjsci@utq.edu.iq

التشوهات المظهرية واختلال نسبة الجنس في الأسماك الذهبية Carassius auratus كموئشر حيوي للتلوث في هور الحمار - جنوب العراق

أياد حنتوش داوود الخفاجي هـى حسن خريبط الخيون "

جامعة البصرة- كلية العلوم ـ قسم علوم الحياة

*E-mail: Hudaa-hassan@yahoo.com

الخلاصة

تضمنت الدراسة الحالية تحديد التشوهات المظهرية والاضطراب في نسبة الجنس للأسماك الذهبية C. auratus الدصطادة من هور الحمار خلا أثنهر الدراسة الممتدة من تشرين الأول 2013 لغاية أيار 2014 بلغ عدد الأسماك المصطادة 106 سمكة ـ تمثلت التشوهات المظهرية بتشوهات العمود

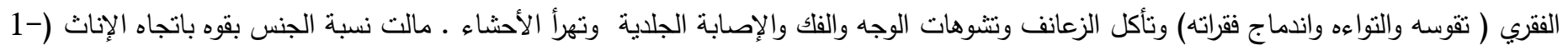

الكلمات المفتاحية: التلوث ، الأسماك الذهبية ، تشوهات العمود الفقري ،هور الحمار •

\title{
The Morphological Deformations and Discrepancy in Sex Ratio in Goldfish (Carassius Auratus) As Biomarker for Pollution in Al- Hammar Marshes - South Iraq
}

\author{
Huda Hassan Khraibet Al-Kayon
}

Ayad Hantoosh Dawood Al- Khafaji

\section{Summary}

The present study include determine the morphological anomalies and sex ratio disruption for gold fish Carassius auratus that captured from Al-Hammar marshes during study period that prolonged from October 2013 to May 2014 . the number of captured fish were 106. Morphological anomalies were represent ell by deformity of vertebral column (curved and fusion of vertebrae ), face and upper jaws, fin erosion, popeye and eye bleeding . Sex ratio tend strongly towed females $(1 ; 25.5)$.

Key words: pollution, Carassius auratus, deformity of vertebral column and Al-Hammar marshes.

استجابات حياتية مبكرة (Suter , 1990)، تكون مهمة لأنها تتكامل

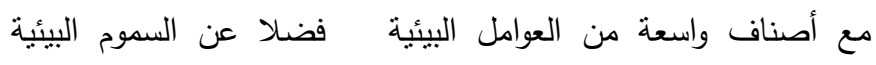
Ecotoxicology

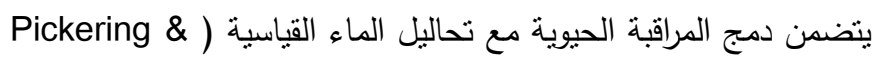
(Pottinger , 1995 لها أثرا كبيرا على الأسماك ، هنشمل:( الهيدروكاربونات النفطية

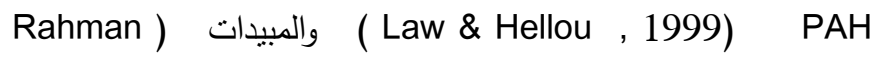

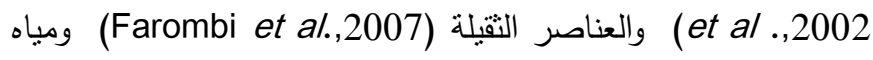
Ortolan ) الصرف الصحي (Parnell,2003) وفضلات المستشفيات (P) (Pنيات

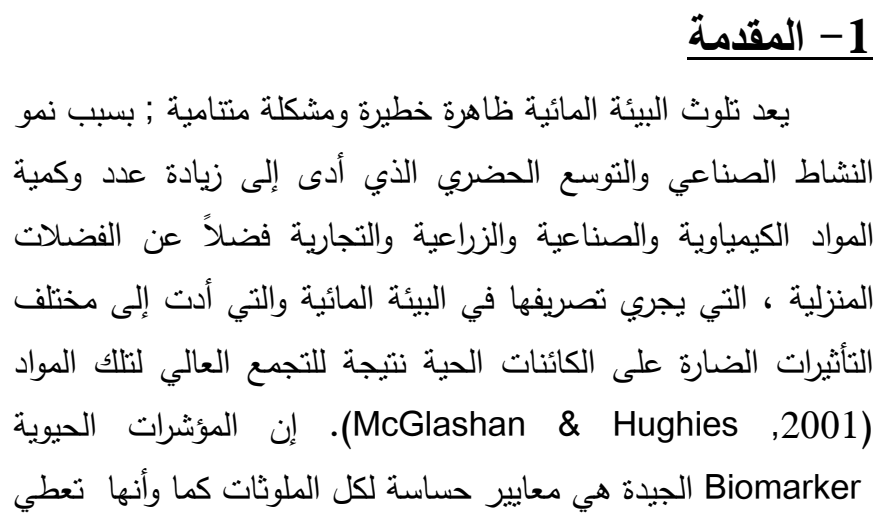


بعد الصيد حفظت الأسماك مباشرةً في حاويات فلينية تحتوي على الثلج المجروش قبل نقلها إلى المختبر.نم قياس العوامل البيئة المتضمنة فئنة

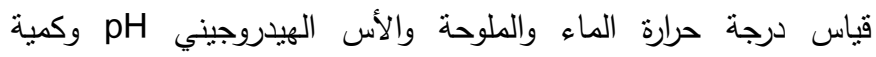
Winkler الأوكسجين المذاب في الماء باستخدام طريقة ونكلر المحورة Modified method البيانات المتعلقة بالعوامل البيئية في جدول رقم (1)الملحق بالبحث.

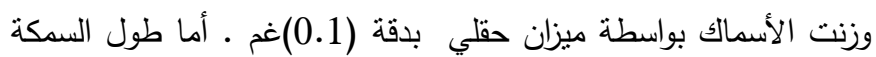
الكلي قد قيس بواسطة مسطرة معلمة وبدقة ( 0.1 سم ). جرى معاينة الأسماك من الناحية الخارجية لتسجيل جميع الملاحظات اللافتة للنظر

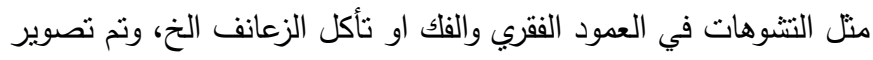

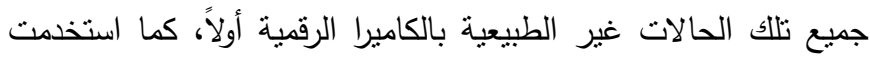
آلية التصوير الإشعاعي لت لتشخيص النتوهات في الجهاز

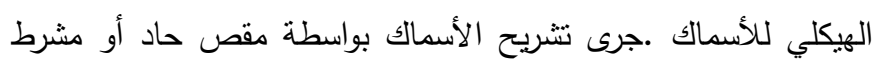

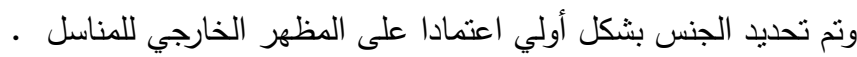

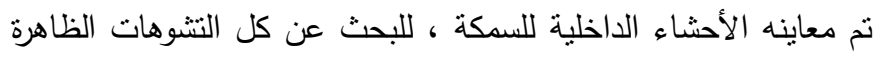
للعيان وتوثيقها بالصور الفوتوغرافية .استقطت عينات نسيجية صغيرة بحجم( 0.5 )سمץ تقريبا ممثلة للإصابات الجلدية وذللك لتبيان الاضطرابات النسيجية المصاحبة.وضعت جميع الأنسجة المنتقاة في لئل

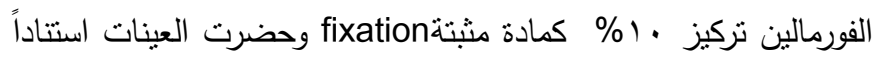

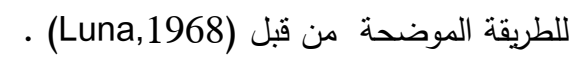
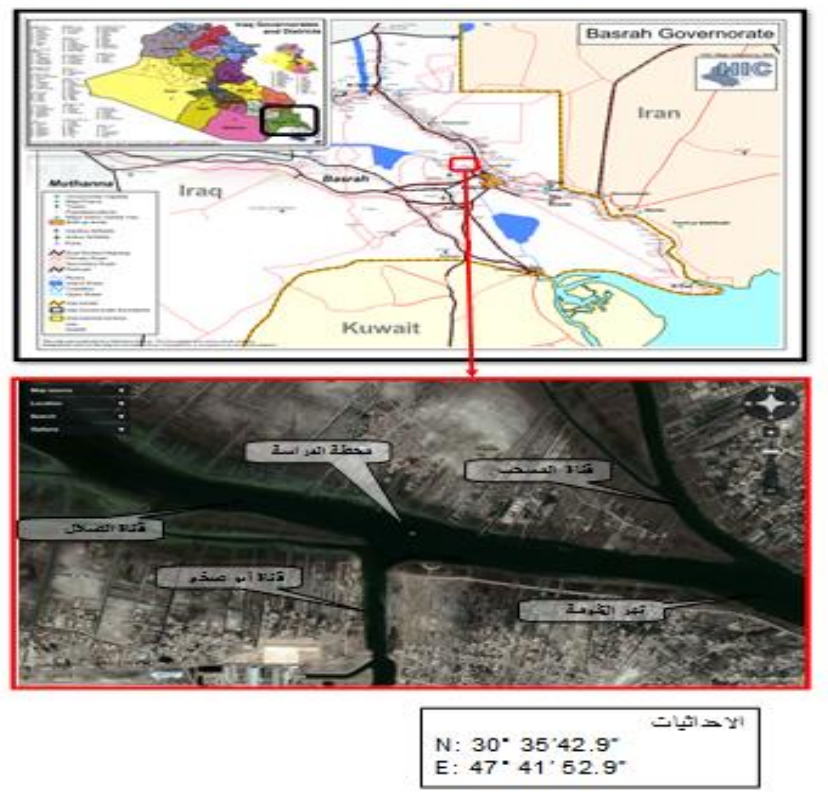

شكل (1) موقع جمع العينات
Ayub , 2007 \& ). من ناحية أخرى تلعب الأهوار الجنوبية دوراً بيئياً

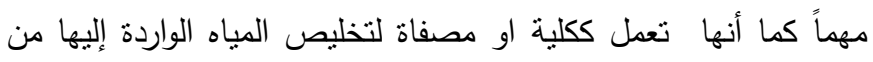

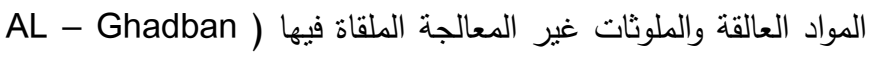

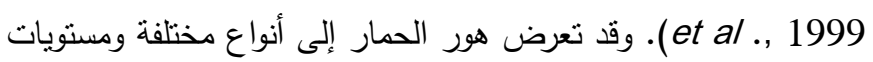

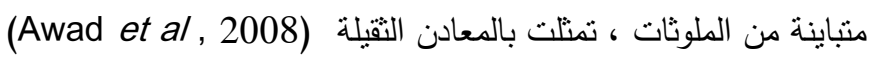

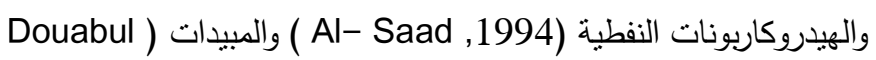

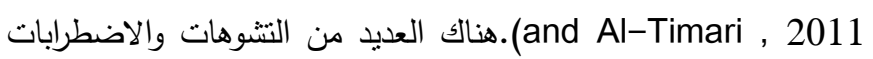
في الأسماك نستخدم كمؤشرات حيوية للتلوث . تمنتات تلك التشوهات على سبيل المثال تشوهات الجهاز الهيكلي (ICES, 1997) وتأكل

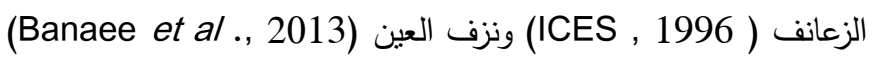

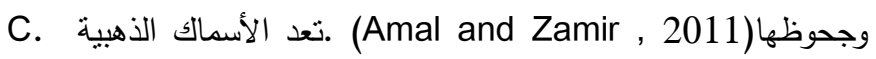
auratus

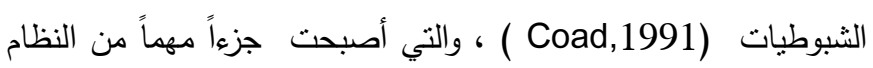
البيئي (Al-Noor,2010).تهدف هذه الدراسة نوضيح العلاقة بين

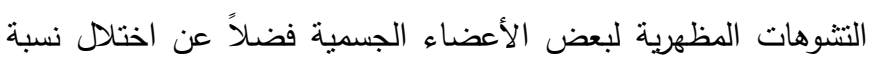

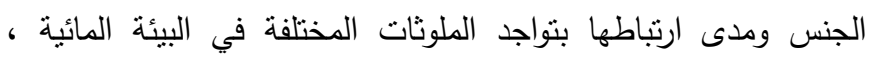
وإمكانية الاعتماد على تلك الاضطرابات كمؤشر لتنيان الأجهادات البيئية

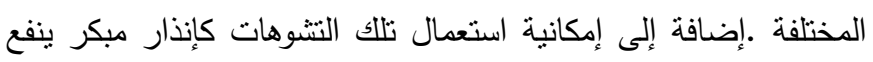
في الكثف عن الملوثات حتى وأن كانت في التزاكيز الدنيا غير المحسوسة ـ كما تلعب الدراسات التكاملية المنطلقة من النعشيق بئ بين

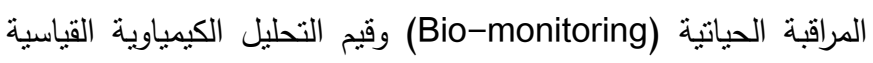
للملوثات.

\section{2- مواد العمل وطرائقه}

يمتد هور الحمار من محافظه ذي قار (جنوب نهر الفرات )في

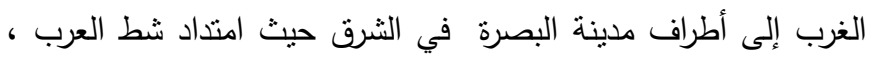

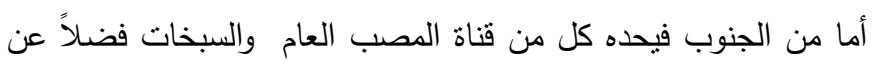

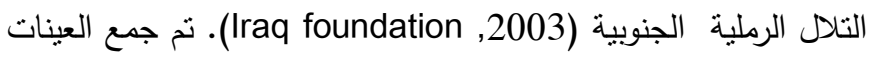

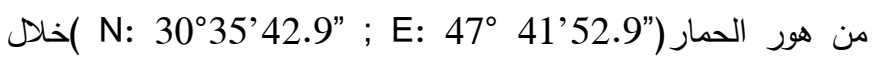

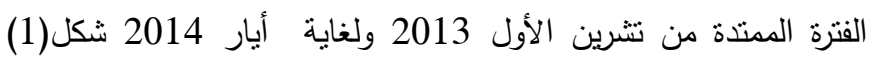
يمثل منطقة جمع العينات ـ وقد استخدمت العديد من الأساليب في جمع لئن

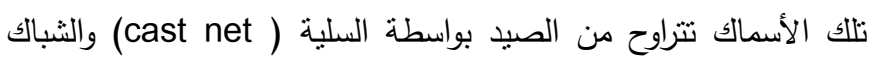
الخيشومية والكرفةSein net ، من ناحية أُخرى جمعت عينات السيطرة من أحواض مركز علوم البحار ، باستعمال شباك السلية. 


\section{3-1-3- تثوهات الجهاز الهيكلي: \\ 1-3-1-3 تثوهات العمود الفقري:}

3- النتائج والمناقشة

3-1-3-التشوهات المظهرية:

تميزت جميع العينات المصطادة خلال فترة الدراسة بظهور التشوهات

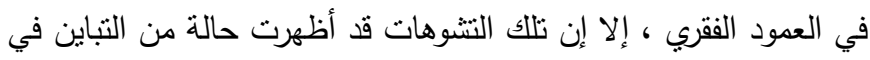
نسب تكرارها (جدول 1). إن أعلى نسبة تكرار (50,40,30\%) قد في لـ

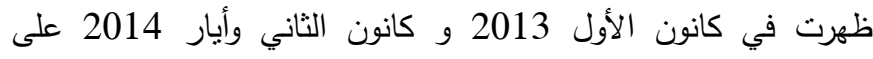

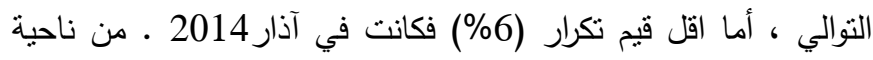

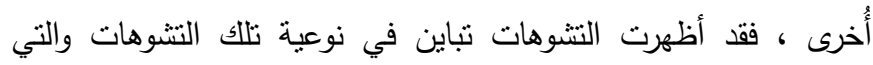

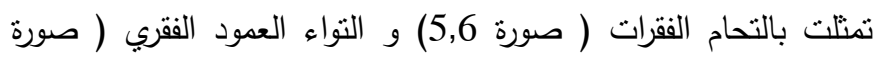

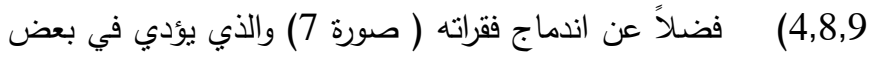
الحالات إلى تقزم السمكة المصحوب باختلال في كتلة السمكة (صورة 10)، وكذلك حدوث ثقوس في العمود الفقري ( صورة 14) .وهناك أراء تبدو منباينة لتفسير تلك الظاهرة ، إلا أنها تلثقي عند نفس النقطة فيما

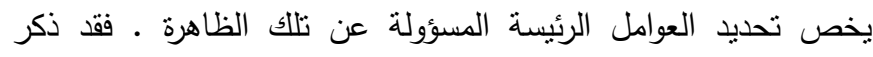
Noga (1996) و Easa(1997) أن العديد من العوامل الوراثية

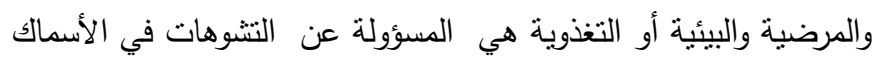

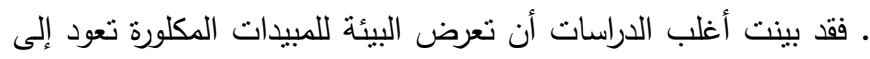
تشوهات واضحة في الجهاز الهيكلي ولاسيما العمود الفقري ( Noga ) ; 1996,العلي ، 2001 ; ال علي ، حين أوضحت دراسة (2004) Abd Elaziz \& Mahamed إن نقص : فيتامين C يؤدي إلى نتوهات في العمود الفقري • 2-3-3-3 لقد بينت النتائج نكرار تأكل الزعانف في

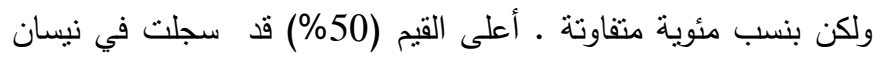

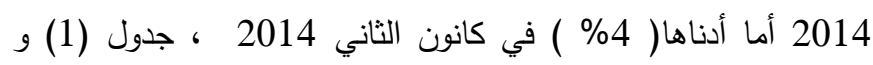

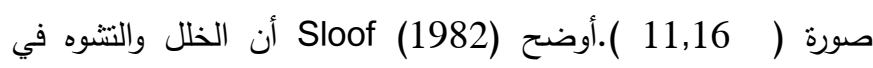
الأشعة الزعنفية مرتبطة ارتباطاً وثيقاً بالبيئة، إذ تعطي معياراً مهماً لتلوث Shawer et (للك البيئة بالعديد من الملوثات ـ من جانب أخر فقد بينة

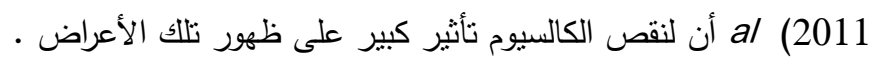

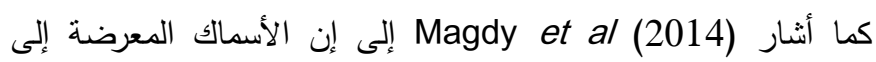
مركبات الدايوكسين تثميز بتاكل زعانفها .

\section{1-1-3 نزف العين:}

تميزت عينات الأسماك المصطادة في شهر تشرين الثاني 2013 و شهري كانون الثاني وآذار 2014 بظهور نسب متفاوتة من فئن

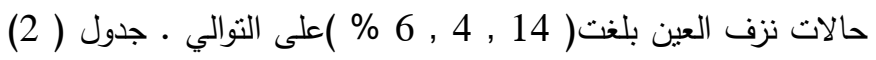
صورة (1) •يعد نزف العين للأسماك من العلامات المهمة التي يستعين

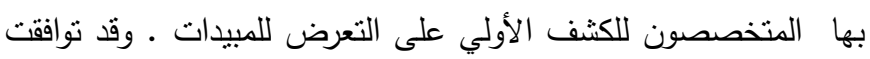

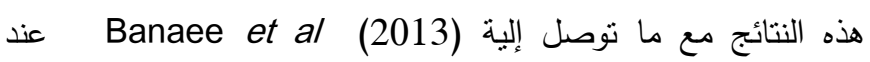

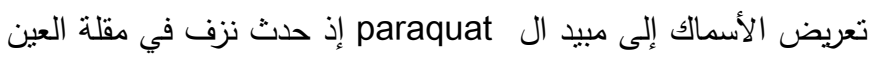

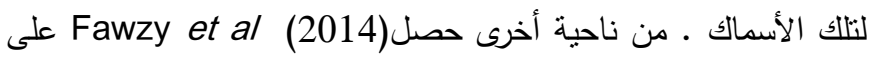
حالة ممانلة في اسماك البلطي أعزاها إلى الإصابة بالبكتريا نوع .Streptococcus 3-3-3 2-3 جحوظ العين: يعتبر جحوظ العينين من الحالات التي نم ملاحظتها في عينات الأسماك المصطادة في شهر كانون الأول 2013 وشباط و آذار 2014 بنسب كانت متفاوتة إذ بلغت(

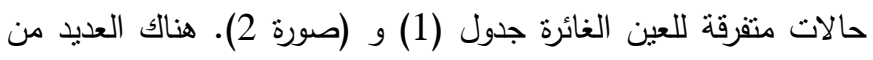
التفسيرات لحالة جحوظ العين في الأسماك ومنها الإصابة ببكتريا Streptococcus

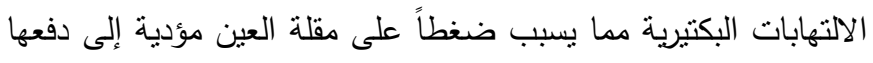

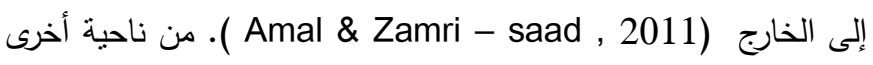
فان إصابة الكلية بيكتريا Renibacterium salmoninarum يعتقد إنها من مسببات الجحوظ في العين (Meyers et al.,2008 ). الجدول (1) النسبة المئوية لظهور النتوهات المظهرية

\begin{tabular}{|c|c|c|c|c|c|c|c|c|}
\hline تلزفنَّفي & القيلين & الأحثَّأ & الألجائدية & كتُكهاك & ألتوهيه & الزعانفل & التقتوبودي & التاريخ \\
\hline & & & & & & & "1 & 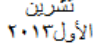 \\
\hline $1 \varepsilon$ & & & & & & $1 \varepsilon$ & $1 \varepsilon$ & 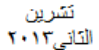 \\
\hline & r. & & & & & & $r$. & الأولوانوب. r \\
\hline$\varepsilon$ & & & & A & $\varepsilon$ & $\varepsilon$ & $\xi$. & الثانيو اكنو. r \\
\hline & $\frac{9}{7}$ & 15 & $\pi$ & $1 \varepsilon$ & $\frac{9}{1.9}$ & 15 & $\frac{1 \varepsilon}{1}$ & 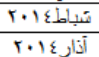 \\
\hline & & ro & $v_{0}$ & & & $\therefore$ & 15 & تنسانع (-r \\
\hline & & & & & & & 0. & F أباري \\
\hline
\end{tabular}


الإصابة الجلدية في أسماك الجري الأفريقية إلى أن الإصابة بيكنة Clarias garipinus)) Pseudomonas ssp. المقاطع النسيجية من الأنسجة المصابة تعاني تغيرات مرضية متل تتكس المقترن بظهور الفجوات وتتخر واستسقاء وكذلك أرتشاح الخلايا الالتهابية في عضلات الأسماك المصابة. جدول (2) يوضح الأمراضيات النسجية في الجلد

\begin{tabular}{|c|c|c|c|c|c|c|}
\hline النتكن & التلبِف & التقجي & التنتخر & الالتهَالايبية & الإنتَستقاء & اللَّاريخ \\
\hline$\varepsilon$. & 7 . & r. & $\gamma \cdot$ & 7 . & $\varepsilon$. & آذارع ا.+r \\
\hline & ro & & & $\infty 0$ & 0. & تبِستانع ا+. \\
\hline
\end{tabular}

3-3 تهرأ الأحشاء :

يعتبر تهرأ أحثاء الأسماك الذي ترافق مع الروائح الكريهة ، إحدى

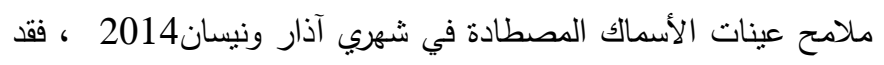
بلغت نسبة الأسماك المعنية (13 , 25\% )على التوالي (جدول الاسطي ) (صورة 20 ) ). فقد سجل الحلفي (2005) نفس النتيجة للأسماك

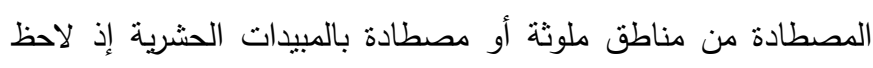

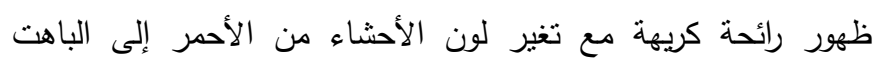
والأخضر من جانب أخر لاحظ الباحث نفسه تعرض الكلاب الصغيرة

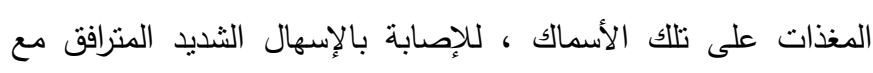
التقئ وهي علامات مؤكدة للتسمم ، وذللك بعد مرور 3ساعات فقط .

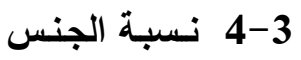

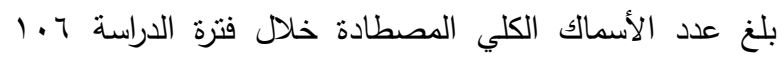
سمكة. تراوح أطوال الأسماك المصطادة بين (12-33) سم،أما أوزانها فقد كانت تتراوح بين (22-626)غم. تم تحديد الجنس للأسماك استتاداً

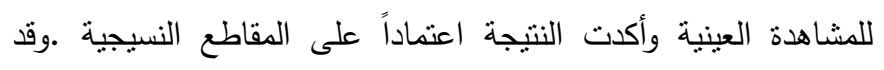

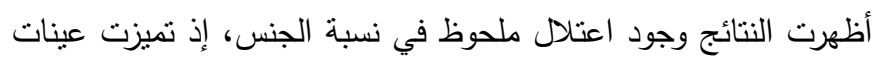

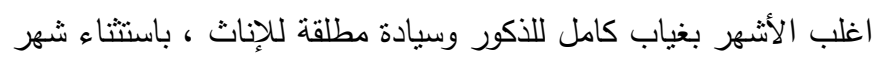

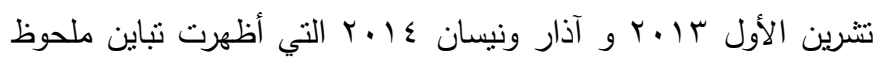

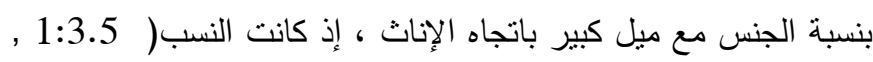

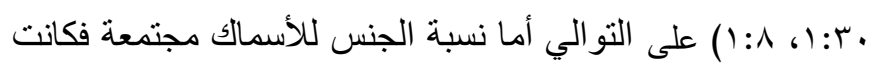

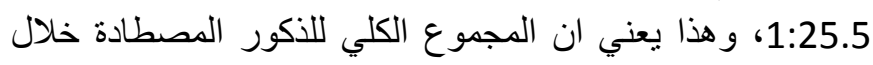

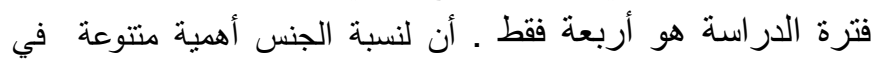

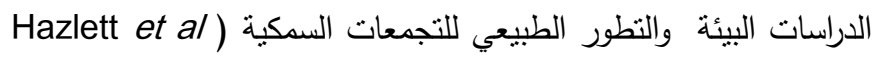

3-3-3-3 تشوهات الوجه والقك:

تميزت عينات الأسماك بظهور نسب متفاوتة لتشوهات الوجه والفاك ،

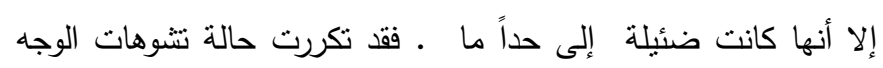

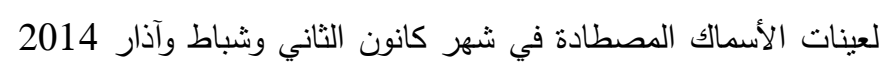

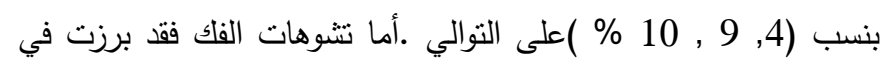

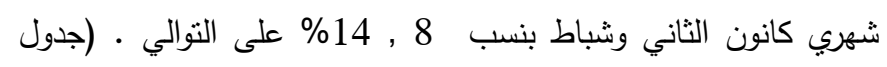

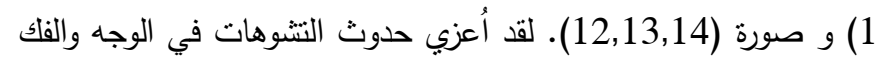

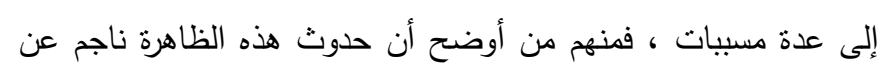

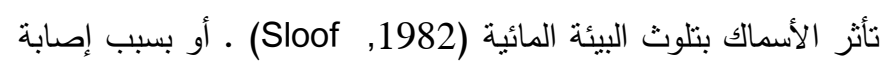
Kocan ) الأسماك بالطفيلي Myxosoma cerebalis - (and Hershberger,2006 3-2 الإصابة الجلدية:

تميزت أثنهر الربيع ( آذار ونيسان ) بظهور إصابات جلدية

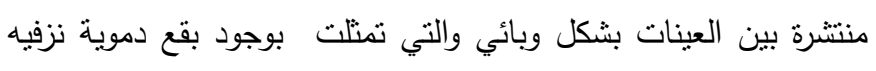

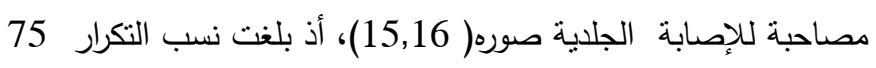

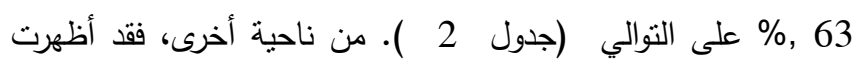

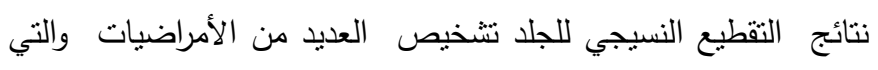

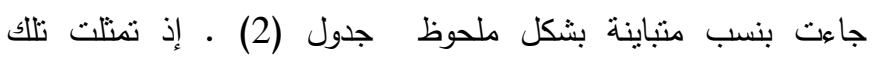

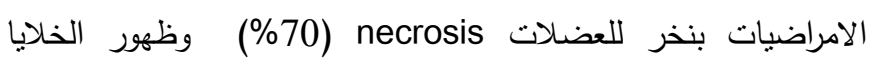
الالتهابية inflamatery cell (60\% ) (صورة 18) فضلا عن التليف

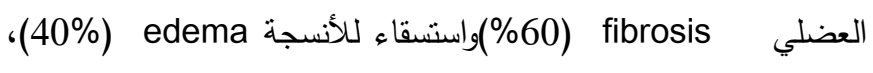
أما تكرار ظاهرة التفجي vaculation فكانت 20\% (صورة 17). كما لوحظ تتكس degeneration (40\%) (صورة 18) و ( جدول2).أن أكثر المسبيات رجحاناً لهذا الوباء هو إصابة الأسماك بالفيروسات فقد

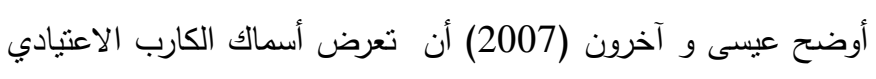
C. carpio

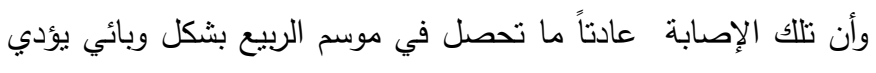

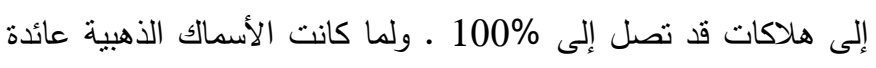

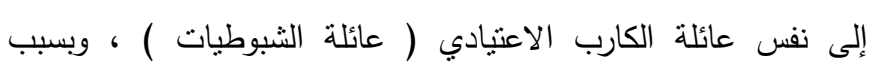

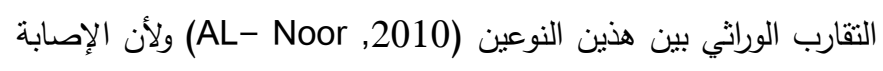
في الدراسة قد حدثت حصراً في أنثهر الربيع ( آذار ونيسان ) ـ ـ واستناداً

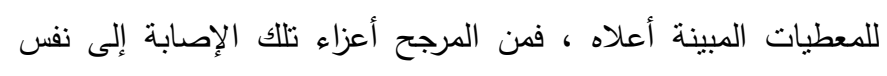

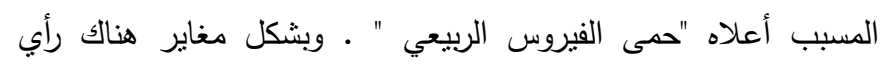

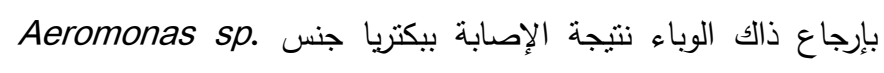

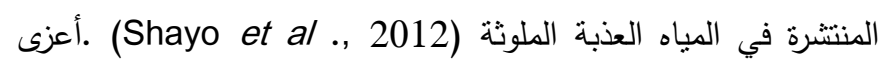




\section{كانون الاول / 1 ـ r}

المجلد •(r)

مجلة علوم ذي قار

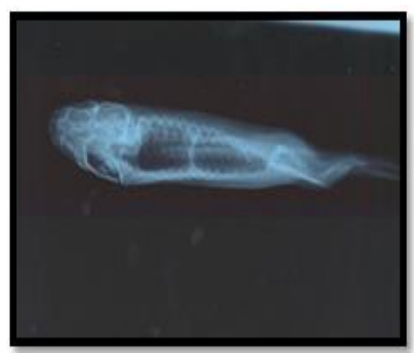

$$
\text { صورة (8) توضح التواء العمود }
$$
الفقري

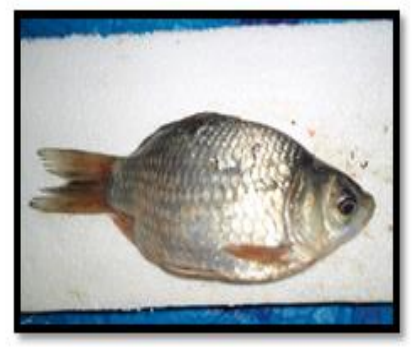

$$
\text { صورة (10) نوضح التقزم في }
$$

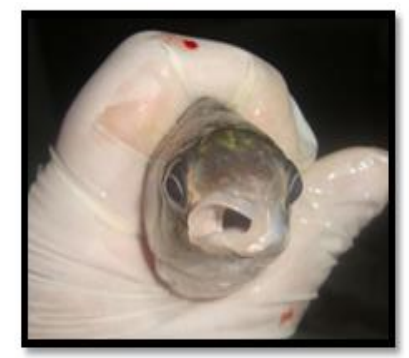

صورة (12) نوضح تشوه الوجه

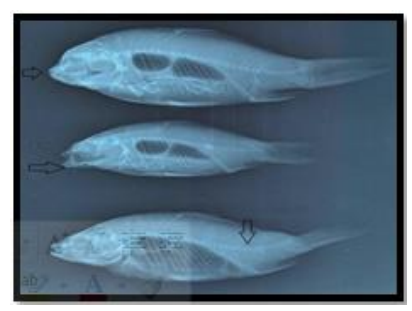

صورة (ع ا) توضح تثوه الفك والوجه والتقوس في العمود الفقريبالاشعة السينية

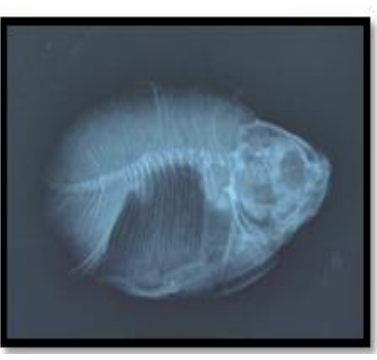

صورة (7) توضح ثقزم

الأسماك بالأشعة السينية

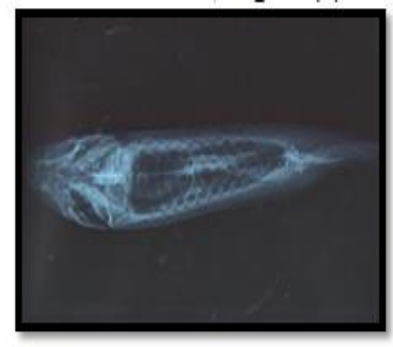

صورة (9) توضح التواء العمود الفقري

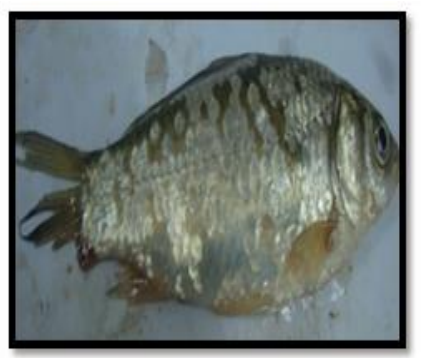

صورة (11) نوضح تأكل الزعانف

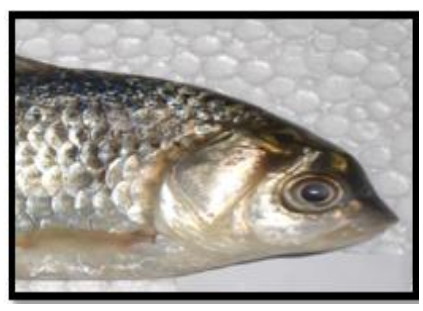

صورة (13) توضح تشوه الفك

2005,.) أن النسبة الطبيعية للجنس في المناطق الأسيوية تكون قريبة

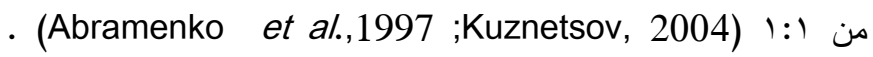
وقد اتفقت نتائج الدراسة الحالية مع ما نوصلت إليه دراسة (eorenzoni

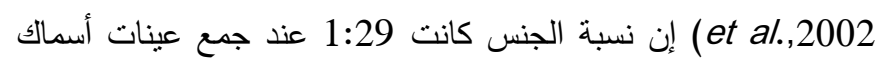
C. auratus الجنس في الحيوانات أهمها اختلاف في نسبة الجنس عند الولادة كنتيجة

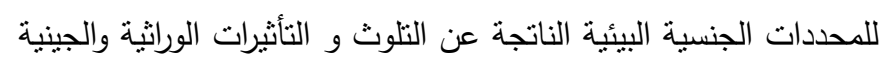

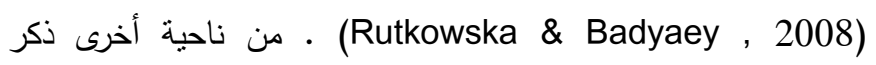
(Hahlbeck,2004)

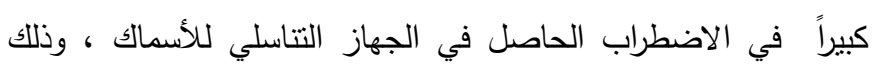

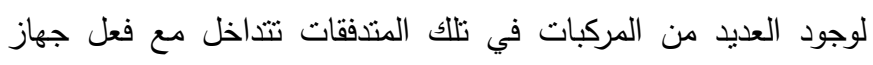
الغدد الصم الأمر الذي يؤدي إلى انحراف نسبة الجنس فضلاً عن فئل التداخل الجنسي في المناسل .

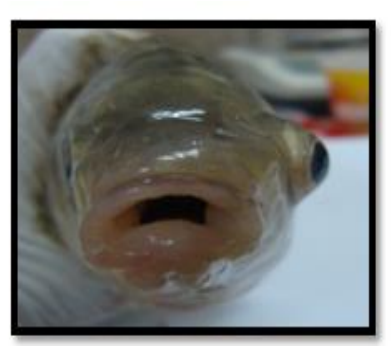

صورة (2) تمثل غور العين

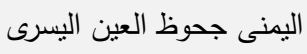

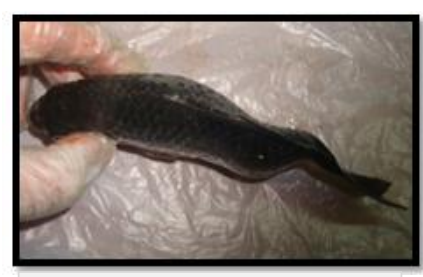

صورة (4) التواء العمود الفقري

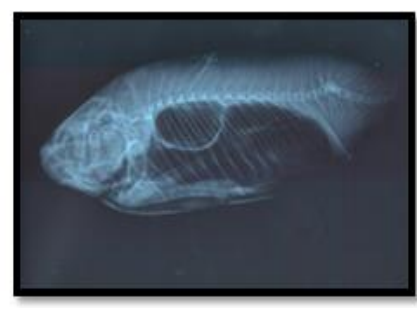

$$
\text { صورة (6) توضح التحام الفقرات }
$$

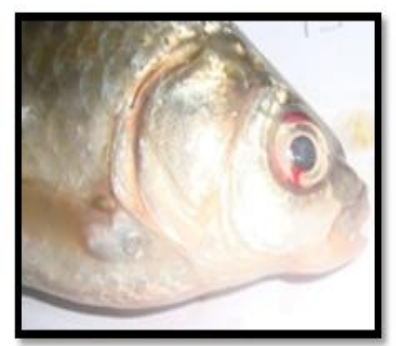

صورة (1) تمثل النزف في العين

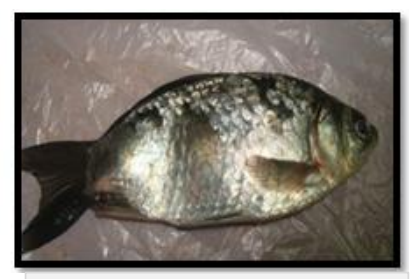

صورة (3) تثوه العمود الفقري

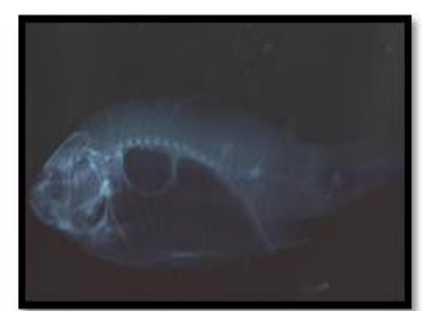

صورة (5) توضح التحام الفقرات بتصوير الأشعة السينية 
References

العلي ، مجدي فيصل مجيد (2004) ـ دراسة بعض المتغيرات الفسلجية

والنسيجية والكيموحيوية في الأسماك الذهبية Carassius auratus الكلوروبيروفوس chlorpyrifos ـ أطروحة دكتوراه ـ كلية العلوم . جامعة البصرة . 182 ص .

الحلفي، مشتاق عبد المهدي عزيز .(2005). أثز المبيدات الحشرية في

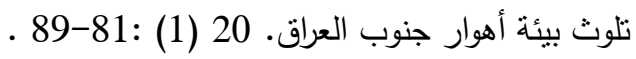
السعدي، أمال غازي ياسر . (2005). دور النبات المائي في الثأثيرات

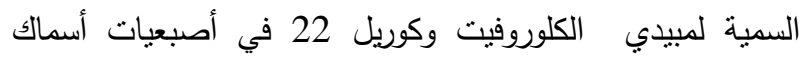

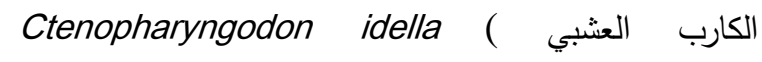
- Valenciennes , 1844)

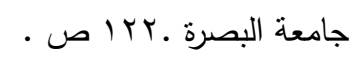

العلي ، بلقيس سهيم عباس(2001) ـ ثأثير العسرة على سمية مبيد

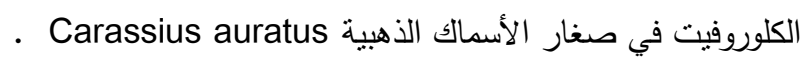

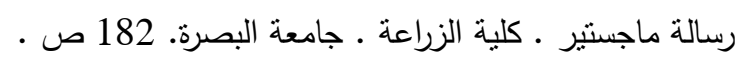

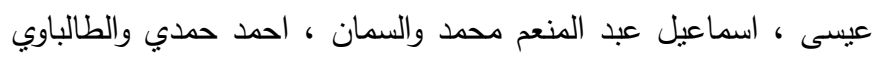

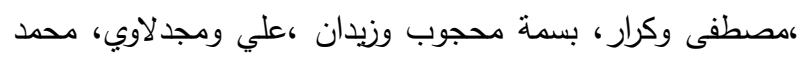

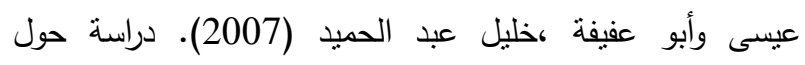
أمراض الأسماك في الوطن العربي ، المنظمه العربية للتنمية

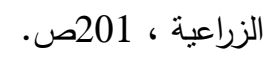

Abd Elaziz, M.A. and Mahamed, M.A.(2004). STUDY ON THE EFFECT OF VITAMIN C DEFICIENCY ON OREOCHROMIS NILOTICUS UNDER INTENSIVE CULTURE SYSTEM. 1rst Ann. Confr. , FVM., Moshtohor, Sept, 2004.

Abramenko, M. I., O. V. Kravchenko, and A. E. Velikoivanenko.(1997). Population genetic structure of the goldfish Carassius auratus gibelio diploid-triploid complex from the Don River Basin. Journal of Ichthyology 37, 56-65.

Al-Ghadban, A. T. ,Saeed, H. A., Al -Dousari, M. , AlHammari, M. and Al-Mutairi, M. (1999). Preliminary assessment of the impact of draining of Iraqi marshes on Kuwats northern marine environment. Part I. Physical manipulation. Water science and Technology 40 (7): 75-87.

Al-Noor, S. S.(2010). Population Status of Gold Fish Carassius auratus in Restored East Hammar Marsh,
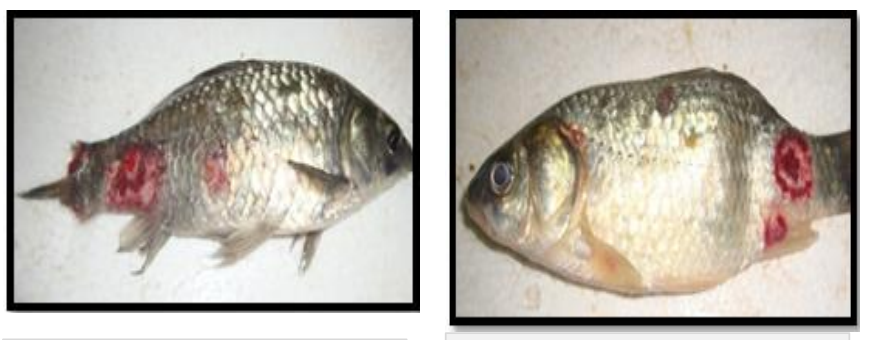

صورة (16) توضح الإصابة

الجلدية وتأكل الزعنفة الننبية

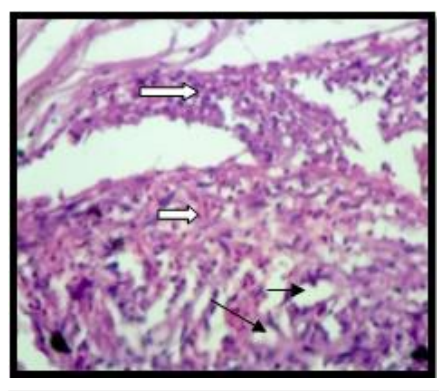

$$
\text { صورة (15) توضح الإصلابة }
$$
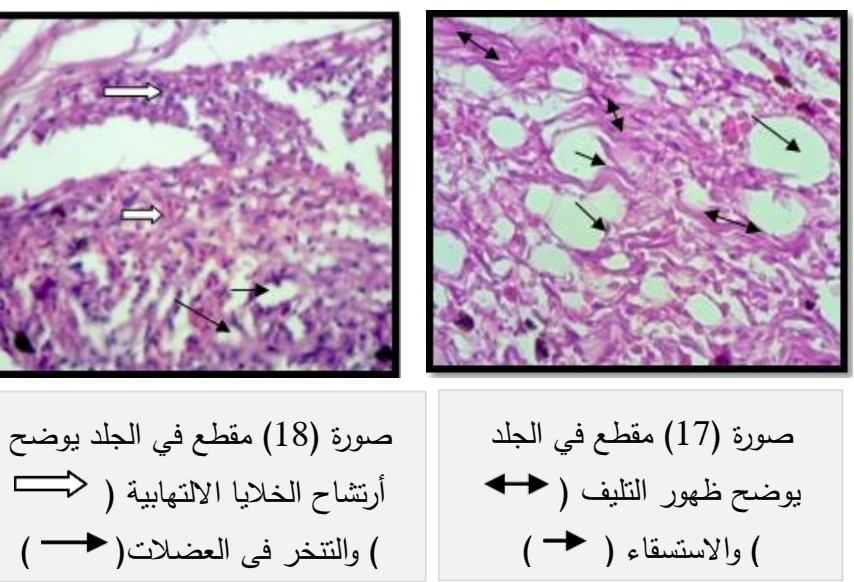

صورة (17) مقطع في الجلد

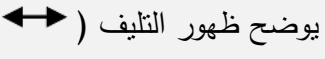

) والاستسقاء ( ) (

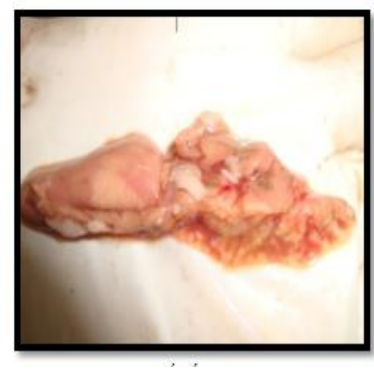

$$
\text { صورة (20) توضح تهرأ }
$$

\begin{tabular}{|c|c|c|c|c|}
\hline الملوحة & الأوكسبلين & الهيدروجيلني & الحرارة & الأناريخ \\
\hline 1.8 & $\frac{V^{\prime}}{4}$ & 1.5 & $r \mu$ & $T .1 T / 1$. \\
\hline$i \lambda$ & $V_{-} T_{1}$ & A & ro & $4.15 / 11$ \\
\hline 5.7 & 1. & $\lambda \varepsilon$ & $1 \varepsilon$ & $T \cdot 1 T / T$ \\
\hline 7.9 & 9.8 & $\lambda .5$ & 17 & $r \cdot \mid \varepsilon / 1$ \\
\hline 0.7 & 7.0 & $\lambda \varepsilon$ & i & $r \cdot I \varepsilon / T$ \\
\hline$r . V$ & $v_{1}$ & NT & Tr & $r \cdot 1 \varepsilon / T$ \\
\hline 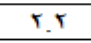 & \%. & 100 & $r 9$ & $r \cdot 1 \varepsilon / \varepsilon$ \\
\hline $1, \varepsilon$ & $\circ$ & $\lambda 1$ & $r 9$ & $r \cdot \mid \varepsilon / 0$ \\
\hline
\end{tabular}

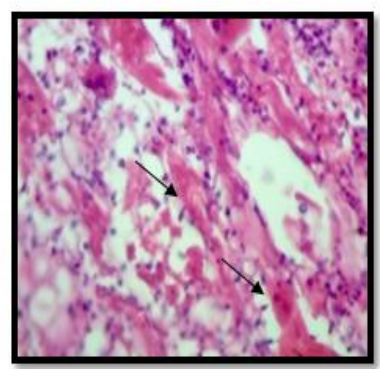

$$
\text { يوضح نتكس العضلات( } 19 \text { (1) مقطع في الجلد }
$$

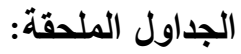
جدول(1) يوضح القياسات البيئية لفترة الدراسة 
Department of Systems Ecology Institute of Applied Environmental Research (ITM) Stockholm University SE-106 91 Stockholm, Sweden.40 PP. edda.hahlbeck@itm.su.se.

Hazlett, B. A., Bach, C. E., Thompson, G. A. \& McLay, C. L. (2005). Movement of male Heterozius rotundifrons (Crustacea: Decapoda: Brachyura) depends upon local sex ratio. New Zealand Journal of Marine and Freshwater Research 39, 157-163.

ICES, 1996. Common diseases and parasites of fish in the North Atlantic: Training guide for identification. ICES Cooperative Research Report, No. 19, pp. 1-27.

ICES, 1997. ICES review of the status of biological effects techniques relative to their potential application programmes. ICES Cooperative Research Report, No. 222, pp. 12-20.

Iraq Foundation (2003) . Physical characteristic of Mesopotamian marshlands of southern Iraq Draft report . 45pp.

Kocan, R. and Hershberger, P. (2006): Differences in Ichthyophonus prevalence and infection severity between upper Yukon River and Tanana River Chinook salmon, Oncorhynchus tsawytscha (Walbaum), stocks. Journal of Fish Diseases 29 (8): 497.

Kuznetsov, V. A. 2004. Changes in the population structure and biological indices of the goldfish Carassius auratus gibelio in the Volga Stretch of the Kuibyshev Reservoir under conditions of intense anthropogenic load on the ecosystem. Journal of Ichthyology 44, 167-174.

Lorenzoni, M., A. J. M. Dorr, R. Erra, G. Giovinazzo, M. Mearelli, and S. Selvi. (2002). Growth and reproduction of largemouth bass (Micropterus salmoides Lace'pe`de, 1802) in Lake Trasimeno (Umbria, Italy). Fisheries Research 56, 89-95.

Luna, L. G. (1968). Manual of Histological Staining Methods of the Armed Forces Institute of Pathology. McGraw-Hill, Inc. Printed, USA, pp: 32-47.

Magdy, I.H. , El-Hady, M. A. , Ahmed, H. A. , A.Elmeadawy,S. and Kenwy, A. M.(2014). A contribution on Pseudomonas aeruginosa infection in African Catfish (Clarias gariepinus). RJPBCS. 5(5): 575-585.

McCarthy,J.F. ,Jimenez, B. D. ; Shugart, L. R. ; Sloop, F. V. ;Oikari, A. (1990): Biological markers in animal sentinels: laboratory studies improve interpretation of fild data. pp . 163-17, In:S.Sandhu
Southern Iraq . JKAU: Mar. Sci., Vol. 21, No. 1, pp: $65-83$.

Al-Saad, H. T. (1994). Distribution of petroleum hydrocarbons in aquatic plant of Hor Al- Hammar marsh Iraq. Marina Mesopotamica 9: 313- 321.

Amal,M.N. and Zamri-Saad,M.(2011). Streptococcosis in Tilapia (Oreochromis niloticus): A Review. Pertanika J. Trop. Agric. Sci. 34 (2): 195 - 206 (2011).

American public health association.(APHA) (2005).Sandard method for the examination of water and waste water, 21st edition . Washington, dc. 1400pp.

Awad, N. A. N., Abdulsahib, H. T. and Jaleel A.A. (2008). Concentration of Trace Metals in Aquatic Plants and sediments of the Southern Maeshes of Iraq (Al-Ahwizah and Al-Hammar).Marsh Bulletin3(1):57-66.

Banaee,M., Davoodi, M. H. and Zoheiri ,F.(2013). Histopathological changes induced by paraquat on some tissues of gourami fish (Trichogaster trichopterus). Open Veterinary Journal, (2013), Vol. 3(1): 36-42.

Coad, B.W. (1991) Fishes of the Tigris-Euphrates Basin; A Critical Check-list. Ichthyology section. Canadian Museum of Nature. Publication, $150 \mathrm{p}$.

DouAbul, A.A. and Al- Timari, A.A.(2011b). Organochlorine pesticide residues in the Mesopotamian southern Iraq and the Arabian Gulf . Absitracts (p10) of conference on development implications with Biodiversity in southern Mesopotamia. Marine Science Center , Basrah University , 12-14 Dec.2011.

Easa, A.I. (1997): Mycotic infection in cultured fish in Egypt . MVSC Suez Canal Univ.

Farombi E. O., Adelowo O. A.and Ajimoko Y. R. (2007). Biomarker of oxidative stress and heavy metal levels as induced by environmental pollution in African cat fish Clarias gariepinus from Nigeria Ogun river. Int. J. Environ. Res. Public Health, 4 (2): 158-165.

Fawzy, N.M., Osman,K.M., Ibrahim,M.E.E., Ali,M.N.M. and Abd- Elrahman,S.S.(2014). Streptococcosis in tilapia: Clinico-pathological picture of experimentally infected tilapia. Life Science Journal 2014;11(9).

Hahlbeck, E. (2004). The juvenile three-spined stickleback - model organism for the study of estrogenic and androgenic endocrine disruption in laboratory and field. Doctoral thesis 2004, 
Suter,G.W.(1990):In McCarthy,J.F. \& Shugart,L.R.(ed),Biomarkers of Environmental Contamination , pp419-428, Lewis Publishers, Boca Raton, FL, USA. (ed) , In Situ Evaluation of Biological Hazards. Plenum Press, N Y .

McGlashan, D.J. and J.M. Hughies, 2001. Genetic evidence for historical continuity between populations of the Australian freshwater fish Craterocephalus stercusmuscarum (Atherinidae) east and west of the Great Diving Range. J. Fish Biol., 59: 55-67.

Meyers,T. ,Burton, T. , Bentz, C. and Starkey,N.(2008).Common diseases of wild and cultured fishes in Alaska. Alaska department of fish and game. $105 \mathrm{pp}$.

Noga, P. (1996): Response of eosinophilic granule cells of Gilthead seabream (Sparusaurata, Teleostei) to bacteria and bacterial products. Cell Tissue Res. Jan. 287 (1): 223 - 30.

Ortolan, M.S. and M.A. Záchia Ayub,( 2007). Cytotoxicity and Genotoxicity of Untreated Hospital Effluents. Brazilian Archives of Biol. Technol., 50(4): 637-643.

Parnell P. (2003). The effects of sewage discharge on water quality and phytoplankton of Hawaiin coastal waters. Marine Environmental Research 55:293-311.

Pickering,A.D.\& Pottinger, T.G.(1995):Biochemical effects of stress. In:Hochachka,P.W.,Mommsen, T.P.(Eds.),Biochemistry \&Molecular Biology of fishes, 5. Environmental and Ecological Biochemistry.Elesvier, Amsterdam, pp.349-379.

Rahman, M.Z., Hossain, Z.M., Ellah, M.F.R., \& Ahmed, G.U. (2002). Effect of Diazinon 60EC on Anabustestudineus, Chama punctatus and barbadesgomonous. Naga. The ICLARM quarterly, 25, 8-11.

Rutkowska, J. \& Badyaev, A. V. (2008). Meiotic drive and sex determination: molecular and cytological mechanisms of sex ratio adjustment in birds. Proceedings of the Royal Society B 363, 16751686. doi: $10.1098 /$ rstb.2007.0006

Shawer,R. , Gomaa,S., Saleh,W. , Soliman, M.K., Khalil, R. and Mona ,S. Z.(2011). Some studies on fish deformity in freshwater fish in Egypt. Life Science Journal, 2011;8(3): 415- 422.

Shayo ,S.D., Mwita, C.J. and Hosea, K. (2012) . Ulcerative Aeromonas Infections in Tilapia (Cichlidae: Tilapiini) from Mtera Hydropower Dam, Tanzania. Open Access Scientific Reports,V0L 1:155.

Sloof, T. S. (1982) : bacterial isolation in marine fish . Japan conf. (7) : (3) : (105-108). 